\title{
Comment on Van de Wiel, Groot and Den Nijs: Gene flow from crop to wild plants and its population-ecological consequences in the context of GM-crop biosafety, including some recent experiences from lettuce
}

\section{Sara Scatasta}

Gene flow from crop to wild plants is one of the potential risks associated with the use of genetically modified organisms. The paper by van de Wiel et al. is an effort to understanding how far we are from answering the question:

"How likely is hybridization from transgenic crops to wild relatives to occur?"

To be able to answer this question is of fundamental importance for the future use of genetically modified organisms in agriculture. The potential evolutionary consequences of the use of transgenic crops have already raised a great deal of concern from consumers as well as producers and the government side of the market for agricultural products. From the consumer side the issues raised can be summarized in 3 broad categories:

1. Loss of biodiversity and environmental concerns;

2. Human health concerns;

3. Concerns related to the effect on prices of agricultural products of an imperfectly competitive biotech industry.

From the producer's point of view the concerns related to gene flow from crop to wild are of 3 main categories:

1. Liability for damages caused to other producers;

2. Ability to identify responsible parties for damages suffered;

3. Ability to calculate the risk of causing damages for which the producer is liable and the extent of these damages and costs of reducing this risk.

From the government point of view the concerns related to gene flow from crop to wild are also of 3 main categories:

1. Ability of setting standards to reduce the risk of damages caused by gene flow;

2. Choice of the optimal governance structure for these standards;

3. Ability to maintain friendly international relations with foreign countries that have adopted different standards.

The paper by van de Wiel et al. analyses the steps that are needed for gene flow from crop to wild to occur actually. In the paper these key elements may influence gene flow:

1. "The presence of wild or weedy relatives in the vicinity of the crop"

2. "The chance of survival of the $F_{1}$ hybrids and their fecundity"

3. "The integration site of the construct on the genome".

\footnotetext{
\# Wageningen University, Social Sciences Group, Wageningen, The Netherlands. E-mail: sara.scatasta@wur.nl
} 
The paper also describes potential barriers to gene flow:

1. Planting a non-transgenic barrier crop around the field planted with the transgenic crop could absorb GM pollen

2. Genetically engineered barriers such as "inhibition of flowering, male sterility, or seed sterility and inserting the transgene into the chloroplast genome".

With respect to lettuce, the paper by van de Wiel et al. describes some new methodologies that can be used to detect whether gene flow has indeed occurred and to what extent.

The paper concludes highlighting the following gaps in the actual knowledge on the issues:

1. The extent to which gene flows from crop to wild occurs in understudied crops such as lettuce;

2. The extent to which gene flows creates 'super weeds';

3. The ecological/evolutionary effects of gene flow.

The paper by van de Wiel et al. certainly confirms where we stand in answering the question asked at the beginning of this brief comment: "How likely is the occurrence of hybridization from transgenic crops to wild relatives?" The paper stresses that hybridization may occur but that further analysis is needed to understand how likely this is. It does, however, contribute greatly to the discussion by describing the steps we need to take now to be able to answer this question in the future.

In order to answer consumers', producers' and governments' concerns about the introduction of genetically modified organisms in European agriculture we need to have a clearer view on what the situation in Europe is with regard to those factors influencing gene flow from crop to wild highlighted in the paper. For example, are there genetically modified crops that are less likely to find wild or weedy relatives in the European Union? As stated by the American Biotechnology Industry Association, "In the United States, most crop species originated elsewhere, so there is very little environmental risk from gene flow for commercial crops such as corn, wheat, soybean, alfalfa, cotton, barley, dry bean and tobacco" (Biotechnology Industry Association 2003). Can we say the same thing for Europe?

Furthermore, is the characteristic mosaic structure of agricultural fields in Europe more likely to cause gene flows from crop to wild than an extensive agricultural-field structure such as that found in the US? When analysing the consequences of genetically modified organisms (GMOs) in European agriculture we need to keep in mind what the advantages of such cropping should be. If the farmer's point of view is taken into consideration, GMOs should increase the profitability of the field. If European farmers are to introduce GMO crops they will lose the premium paid by consumers to have GMO-free products. This means that farmers should be able to produce GMOs at a cost advantage with respect to their international competitors, and this cost advantage should be at least as great as the loss of the premium. The potential liability from damages caused by gene flow from crop to wild introduces a component of risk that should be quantified if the farmer is to base the choice of adoption of GMOs on the correct information.

\section{References}

Biotechnology Industry Association, 2003. Weediness and gene flow. Available: [http://www.bio.org/foodag/background/geneflow.asp] (June 25th, 2003). 